PROCEEDINGS OF THE

AMERICAN MATHEMATICAL SOCIETY

Volume 129, Number 10, Pages 2941-2946

S 0002-9939(01)06134-2

Article electronically published on April 17, 2001

\title{
A NOTE ON THE PERIODIC ORBITS AND TOPOLOGICAL ENTROPY OF GRAPH MAPS
}

\author{
LL. ALSEDÀ, D. JUHER, AND P. MUMBRÚ \\ (Communicated by Michael Handel)
}

\begin{abstract}
This paper deals with the relationship between the periodic orbits of continuous maps on graphs and the topological entropy of the map. We show that the topological entropy of a graph map can be approximated by the entropy of its periodic orbits.
\end{abstract}

\section{INTRODUCTION}

The notion of topological entropy appeared early in the sixties (see [1). It is defined for continuous maps on compact metric spaces and is a quantitative measure of the dynamical complexity of the map. It is an important topological invariant.

There are some properties of the dynamical behavior of the maps which are controlled by the topological entropy. For instance, it measures the exponential growth rate, when $n$ tends to infinity, of the number of different orbits of length $n$ if we use certain precision to distinguish two orbits (see [6]). For a piecewise monotone map $f$ of the interval, it measures also the exponential rate of increase with $n$ of the number of maximal intervals of monotonicity of $f^{n}$ (see [10]).

We are interested in relating periodic orbits and topological entropy. For continuous maps on the interval, to every periodic orbit $P$ of $f$ we can associate a number $h(P)$ which is the topological entropy of the "connect-the-dots" map corresponding to $P$ or the "linearization" of $P$. In fact, this entropy corresponds to the infimum of the entropies of all maps exhibiting orbits with the same combinatorics as $P$ (see Corollary 4.4.7 of [2]).

In the interval case it is possible to show that the entropy of any map $f$ is the supremum of the values $h(P)$ corresponding to all the periodic orbits $P$ of $f$. Furthermore, for each $n$, we can take this supremum only over the orbits of period $k>n$. This result was stated by Takahashi [12] and proved with the assumption that $f$ is piecewise monotone. In the general case it was also proved in an independent way by Block and Coven [5] and Misiurewicz and Nitecki [9].

Since the topological entropy is usually considered as a measure of the degree of chaos, a natural problem is developing algorithms for calculating it (see 7], [11] or [4]). These algorithms are based on different properties of the entropy and some of

Received by the editors February 10, 2000.

2000 Mathematics Subject Classification. Primary 37E25, 37B40; Secondary 54H20, 54C70.

Key words and phrases. Graph maps, periodic orbits, topological entropy.

The authors have been partially supported by the DGES grant number PB96-1153 and the INTAS OPEN 97 grant number 97-1843. 
them take into account the existence of periodic orbits (see, for instance, [5]) and, in particular, properties like Takahashi's result.

In this paper we show that an analogous relation between periodic behavior and topological entropy is satisfied for continuous maps on graphs. It has been motivated by a question posed by S. Kolyada and N. Snoha to the first author. To this end we introduce some basic notation and state in detail the main result of the paper.

A (finite) graph $G$ is a compact connected Hausdorff space which contains a finite non-empty set $V$ (the set of vertices), such that every connected component of $G \backslash V$ is homeomorphic to an open interval of the real line. These connected components are called edges. Given a point $x \in V$, the number of edges whose boundaries contain $x$ (with the edges whose closures are homeomorphic to a circle counted twice) will be called the valence of $x$. We will denote the set of points of valence larger than 2 by $B(G)$ (the set of branching points). Since any graph can be embedded in $\mathbb{R}^{3}$, in what follows we will consider each graph endowed with the topology induced by the topology of $\mathbb{R}^{3}$.

We shall call a set $J \subset G$ an interval if there is a homeomorphism $\phi: I \longrightarrow J$, where $I$ is $[0,1],(0,1],[0,1)$ or $(0,1)$, and there are no vertices in $J$ except perhaps $\phi(0)$ and $\phi(1)$. The set $\phi((0,1))$ will be called the interior of $J$ and will be denoted $\operatorname{Int}(J)$. If $I=[0,1]$, the interval $J$ will be called closed and if $I=(0,1)$, the interval $J$ will be called open. Notice that it may happen that $\operatorname{Int}(J) \neq J$ for an interval $J$ being an open set in the topology of $G$. For example, let $G$ be a graph with two vertices and one edge. Then $G$ is an interval and an open set as a topological space but $\operatorname{Int}(G)$ does not contain the vertices. As usual, a subinterval of an interval $J$ will be an interval contained in $J$.

A continuous map $f$ from a graph $G$ into itself is called a graph map. Let $f$ be a graph map and let $A$ be a finite set such that $f(A) \subset A$. We define an equivalence relation among the triplets $(G, A, f)$ as follows: $(G, A, f)$ and $\left(G^{\prime}, A^{\prime}, f^{\prime}\right)$ are equivalent if there exists a homeomorphism $\phi: G \longrightarrow G^{\prime}$ with $\phi(A)=A^{\prime}$ such that $f$ and $\phi^{-1} \circ f^{\prime} \circ \phi$ are homotopic relative to $A$. Notice that then $\left.\phi^{-1} \circ f^{\prime} \circ \phi\right|_{A}=\left.f\right|_{A}$. Each equivalence class of this relation, denoted by $[G, A, f]$, will be called an action.

We define the entropy of $[G, A, f]$, denoted $h([G, A, f])$, as

$$
h([G, A, f])=\inf \left\{h\left(f^{\prime}\right):\left(G^{\prime}, A^{\prime}, f^{\prime}\right) \in[G, A, f]\right\} .
$$

Given an action $[G, A, f]$, from [3] it follows that there exists a representative $(G, A, g)$ of $[G, A, f]$ that gives the entropy of the action, i.e., such that $h(g)=$ $h([G, A, f])$. We shall use this fact to obtain lower bounds of the topological entropy of a graph map. For a finite set $P$ we denote its cardinality by $|P|$. The main result of the paper is the following.

Theorem 1.1. Let $G$ be a graph and let $f: G \longrightarrow G$ be a graph map. For each nonnegative integer $m$ we have

$$
h(f)=\sup \{h([G, P, f]): P \text { periodic orbit of } f \text { and }|P|>m\} .
$$

Our proof of this result is based in the main ideas used in the proof of the analogous result for interval maps (see, for instance, Theorem 4.4.10 of [2]) and in some properties of graph maps pointed out in [8]. 


\section{Proof of Theorem 1.1}

We shall need a simple property which is well known for interval and circle maps (see, for instance, [2]).

Lemma 2.1. Let $f: G \longrightarrow G$ be a graph map. Let $\left\{J_{i} \subset G: i=1,2, \ldots, n\right\}$ be a family of closed intervals such that $f\left(J_{i}\right) \supset J_{i+1}$ for $i=1,2, \ldots, n-1$, and $f\left(J_{n}\right) \supset J_{1}$. Then there exists a point $x=f^{n}(x)$ such that $f^{i}(x) \in J_{i+1}$ for all $0 \leq i \leq n-1$.

Proof. Since $f\left(J_{n}\right) \supset J_{1}$ and there are no vertices in the interior of an interval, there exists a closed subinterval $K_{n} \subset J_{n}$ with $f\left(K_{n}\right)=J_{1}$. Analogously, since $f\left(J_{n-1}\right) \supset J_{n}$, there is a closed subinterval $K_{n-1} \subset J_{n-1}$ with $f\left(K_{n-1}\right) \subset J_{n}$ and $f^{2}\left(K_{n-1}\right)=J_{1}$.

Inductively, there is a closed subinterval $K_{1} \subset J_{1}$ with $f^{i}\left(K_{1}\right) \subset J_{i+1}$, for $i=1,2, \ldots, n-1$, and $f^{n}\left(K_{1}\right)=J_{1}$. Then, since $f^{n}$ is a continuous map and there are no vertices in $\operatorname{Int}\left(J_{1}\right)$, the intermediate value theorem ensures the existence of a point $x \in K_{1}$ such that $f^{n}(x)=x$. By the election of $K_{1}$ it follows that $f^{i}(x) \in J_{i+1}$ for all $0 \leq i \leq n-1$ and the lemma follows.

As for interval and circle maps, an important notion for obtaining minimal models of an action is the notion of local monotonicity. Now we introduce the corresponding definition for graph maps. Let $I$ be a closed interval of a graph $G$. Let $f: I \longrightarrow G$ be a graph map and let $x \in \operatorname{Int}(I)$. We denote by $K(x)$ the connected component of $f^{-1}(f(x))$ containing $x$. We say that $f$ is locally monotone at $x$ if there exists an open neighborhood $U$ of $K(x)$ such that $f(U)$ is homeomorphic to an interval of the real line (perhaps degenerate to a point) and $\left.f\right|_{U}$ is monotone (not necessarily strictly) as an interval map. We say that $f$ is monotone if it is locally monotone at each point of $I$.

Let $(G, A, f)$ be a representative of an action. We say that $(G, A, f)$ is monotone if $f$ restricted to any interval $I$ without points of $A \cup B(G)$ in its interior is monotone. If in addition $f(B(G)) \subset A \cup B(G)$, then $(G, A, f)$ is called simplicial. Given an action, as we noticed above, there is a representative such that its entropy coincides with the topological entropy of the action. Moreover, in [3] it is shown that this representative can be taken to be simplicial. We shall use this fact in order to prove that the existence of a horseshoe gives a lower bound of the topological entropy of the map. To this end we introduce the notion of horseshoe.

Let $s \geq 2$. An $s$-horseshoe for $f$ is a closed interval $I \subset G$ and closed subintervals $J_{1}, J_{2}, \ldots, J_{s}$ of $I$ with pairwise disjoint interiors, such that $f\left(J_{i}\right)=I$ for $j=$ $1,2, \ldots, s$. An $s$-horseshoe is strong if in addition the intervals $J_{1}, J_{2}, \ldots, J_{s}$ are contained in $\operatorname{Int}(I)$ and are pairwise disjoint.

Proposition 2.2. Let $f: G \longrightarrow G$ be a graph map. Assume that $f^{k}$ has a strong $s$-horseshoe for some $k \geq 1$ and $s \geq 3$. Then there is a periodic orbit $P$ of $f$ with period $|P| \geq 2(s-2)$ such that $h([G, P, f]) \geq \frac{1}{k} \log (s-2)$.

Proof. From the definition of a strong horseshoe there exist a closed interval $I \subset G$ and pairwise disjoint subintervals $J_{1}, J_{2}, \ldots, J_{s}$ contained in the interior of $I$, such that $f^{k}\left(J_{i}\right)=I$ for $i=1,2, \ldots, s$. Thus we have $f^{k}\left(J_{1}\right) \supset J_{i}, f^{k}\left(J_{i}\right) \supset J_{1}$, $f^{k}\left(J_{s}\right) \supset J_{i}$ and $f^{k}\left(J_{i}\right) \supset J_{s}$ for $i=2,3, \ldots, s-1$. 
Then we consider the sequence of intervals $\left\{I_{i}: i=1,2, \ldots, 4(s-2)\right\}$ defined by

$$
I_{i}=\left\{\begin{array}{lll}
J_{j+1} & \text { if } i=2 j-1 & \text { for } j=1,2, \ldots, s-2, \\
J_{1} & \text { if } i=2 j & \text { for } j=1,2, \ldots, s-2, \\
J_{j+1-(s-2)} & \text { if } i=2 j-1 & \text { for } j=s-1, s, \ldots, 2(s-2), \\
J_{s} & \text { if } i=2 j & \text { for } j=s-1, s, \ldots, 2(s-2) .
\end{array}\right.
$$

Actually, this sequence is

$$
J_{2}, J_{1}, J_{3}, J_{1}, \ldots, J_{1}, J_{s-1}, J_{1}, J_{2}, J_{s}, J_{3}, J_{s}, \ldots, J_{s}, J_{s-1}, J_{s} \text {. }
$$

It satisfies the hypothesis of Lemma 2.1 for $f^{k}$. Thus, there exists a periodic orbit $Q=\left\{x, f^{k}(x), f^{2 k}(x), \ldots\right\}$ of $f^{k}$ with $f^{i k}(x) \in I_{i+1}$. Let $P$ be the periodic orbit of $f$ containing $Q$. Obviously $|P| \geq|Q|$. Furthermore $|Q| \geq 2(s-2)$. Indeed, if $|Q|<2(s-2)$, then there exists $i \in\{1,3,4, \ldots, s-1\}$ with $x \in J_{i} \cap J_{2}$, which gives a contradiction.

Given $(\bar{G}, \bar{P}, \bar{f}) \in[G, P, f]$, if $\phi$ is the homeomorphism given by the equivalence between $(\bar{G}, \bar{P}, \bar{f})$ and $(G, P, f)$, then the subset $\phi(Q)$ of $\bar{P}$ satisfies $\left(\bar{G}, \phi(Q), \bar{f}^{k}\right) \in$ $\left[G, Q, f^{k}\right]$. Since $\frac{1}{k} h\left(\bar{f}^{k}\right)=h(\bar{f})$ we have

$$
\begin{aligned}
h([G, P, f]) & =\inf \{h(\bar{f}):(\bar{G}, \bar{P}, \bar{f}) \in[G, P, f]\} \\
& =\inf \left\{\frac{1}{k} h\left(\bar{f}^{k}\right):(\bar{G}, \bar{P}, \bar{f}) \in[G, P, f]\right\} \\
& \geq \inf \left\{\frac{1}{k} h(\bar{f}):(\bar{G}, \bar{Q}, \bar{f}) \in\left[G, Q, f^{k}\right]\right\}=\frac{1}{k} h\left(\left[G, Q, f^{k}\right]\right) .
\end{aligned}
$$

Now, we prove that $h\left(\left[G, Q, f^{k}\right]\right) \geq \log (s-2)$ and we are done. From 3 there exists a simplicial representative $(G, Q, g)$ of $\left[G, Q, f^{k}\right]$ such that $\left.f^{k}\right|_{Q}=\left.g\right|_{Q}$ and $h\left(\left[G, Q, f^{k}\right]\right)=h(g)$. Notice that $g$ has a strong $(s-2)$-horseshoe. Indeed, for each $i \in\{2,3, \ldots, s-1\}$ there is an element from $Q \cap J_{i}$ mapped to $J_{1}$ and an element from $Q \cap J_{i}$ mapped to $J_{s}$. Thus, since $g$ is $Q$-monotone and $J_{i} \subset I$, every $g\left(J_{i}\right)$ contains all the intervals $J_{2}, J_{3}, \ldots, J_{s-1}$. Thus $g$ also has a strong $(s-2)$-horseshoe as we claimed because $J_{1}, J_{2}, \ldots, J_{s}$ is a strong $s$-horseshoe of $f^{k}$.

From this fact and Lemma 3.4 of $[8$ it follows that $h(g) \geq \log (s-2)$ and

$$
h([G, P, f]) \geq \frac{1}{k} h\left(\left[G, Q, f^{k}\right]\right)=\frac{1}{k} h(g) \geq \frac{1}{k} \log (s-2) .
$$

This ends the proof.

Lastly we prove Theorem 1.1.

Proof of Theorem 1.1. From the definition of $h([G, P, f])$ it follows that

$$
h(f) \geq \sup \{h([G, P, f]): P \text { periodic orbit of } f \text { and }|P|>m\} .
$$

So, we shall prove the other inequality.

If $h(f)=0$ we are done. So we assume that $h(f)>0$. From Theorem B of [8], there are sequences of natural numbers $\left(k_{n}\right)_{n=1}^{\infty}$ and $\left(s_{n}\right)_{n=1}^{\infty}$ such that $f^{k_{n}}$ has an $s_{n}$-horseshoe for each $n \geq 1$ and $h(f)=\lim \sup _{n \rightarrow \infty} \frac{1}{k_{n}} \log s_{n}$. Furthermore, from Lemma 3.3 of [8], it follows that $f^{k_{n}}$ has a strong $\left(s_{n}-2\right)$-horseshoe for each $n \geq 1$.

Now we distinguish two cases.

Case 1. $h(f)=\infty$.

Then the natural numbers $s_{n}$ take infinitely many different values. So we can choose them in such a way that $\left(s_{n}\right)_{n=1}^{\infty}$ is an increasing sequence. 
From Proposition[2.2 (with $s=s_{n}-2$ ) it follows that for each $n \in \mathbb{N}$ there exists a periodic orbit $P_{n}$ of $f$ such that $\left|P_{n}\right| \geq 2\left(s_{n}-4\right)$ and $h\left(\left[G, P_{n}, f\right]\right) \geq \frac{1}{k_{n}} \log \left(s_{n}-4\right)$. Given any $M>0$, since $\lim \sup _{n \rightarrow \infty} \frac{1}{k_{n}} \log s_{n}=\infty$, we can choose an $n$ such that $\frac{1}{k_{n}} \log \left(s_{n}-4\right)>M$ and $2\left(s_{n}-4\right)>m$. Then we have $\left|P_{n}\right|>m$ and

$$
h\left(\left[G, P_{n}, f\right]\right) \geq \frac{1}{k_{n}} \log \left(s_{n}-4\right)>M .
$$

Since $M$ is arbitrary, it follows that

$$
h(f)=\infty=\sup \{h([G, P, f]): P \text { periodic orbit of } f \text { and }|P|>m\},
$$

as we claimed.

Case 2. $0<h(f)<\infty$.

Since $\left(k_{n}\right)_{n=1}^{\infty}$ and $\left(s_{n}\right)_{n=1}^{\infty}$ are sequences of natural numbers and

$$
0<\limsup _{n \rightarrow \infty} \frac{1}{k_{n}} \log s_{n}<\infty,
$$

then either both sequences $\left(k_{n}\right)_{n=1}^{\infty}$ and $\left(s_{n}\right)_{n=1}^{\infty}$ take infinitely many different values or both sequences take finitely many values.

If $\left(k_{n}\right)_{n=1}^{\infty}$ and $\left(s_{n}\right)_{n=1}^{\infty}$ take infinitely many different values, we can choose the sequence $\left(s_{n}\right)_{n=1}^{\infty}$ in such a way that it is an increasing sequence. As in Case 1, for each $n \in \mathbb{N}$ there exists a periodic orbit $P_{n}$ of $f$ such that $\left|P_{n}\right| \geq 2\left(s_{n}-4\right)$ and $h\left(\left[G, P_{n}, f\right]\right) \geq \frac{1}{k_{n}} \log \left(s_{n}-4\right)$. Now $\lim \sup _{n \rightarrow \infty} \frac{1}{k_{n}} \log \left(s_{n}-4\right)=h(f)$. Thus, given any $\epsilon>0$, there exists an $n$ such that $\frac{1}{k_{n}} \log \left(s_{n}-4\right)>h(f)-\epsilon$ and $2\left(s_{n}-4\right)>m$. Then we have $\left|P_{n}\right|>m$ and

$$
h\left(\left[G, P_{n}, f\right]\right) \geq \frac{1}{k_{n}} \log \left(s_{n}-4\right)>h(f)-\epsilon .
$$

Since $\epsilon$ is arbitrary, it follows that $\sup \{h([G, P, f]):|P|>m\} \geq h(f)$, as we claimed.

If $\left(k_{n}\right)_{n=1}^{\infty}$ and $\left(s_{n}\right)_{n=1}^{\infty}$ take finitely many different values, then there exist natural numbers $k$ and $s$ such that $h(f)=\frac{1}{k} \log s$ and $f^{k}$ has an $s$-horseshoe. Then we can take $k_{n}=n k$ and $s_{n}=s^{n}$ for all $n \geq 1$ and we have $\lim \sup _{n \rightarrow \infty} \frac{1}{k_{n}} \log s_{n}=$ $h(f)$. Also, from Lemmas 3.2 and 3.3 of [8], it follows that $f^{n k}$ has an $s^{n}$-horseshoe and consequently a strong $\left(s^{n}-2\right)$-horseshoe, for each $n \geq 1$. The rest of the proof follows as above.

\section{REFERENCES}

1. R. Adler, A. Konheim and M. McAndrew, Topological entropy, Trans. Am. Math. Soc. 114 (1965), 309-319. MR 30:5291

2. Ll. Alsedà, J. Llibre and M. Misiurewicz, Combinatorial Dynamics and entropy in dimension one, Advanced Series in Nonlinear Dynamics 5, World Scientific, Singapore, 1993. MR 95j:58042

3. Ll. Alsedà, F. Mañosas and P. Mumbrú, Minimizing topological entropy for continuous maps on graphs, Ergod. Th. \& Dynam. Sys. 20 (2000), 1559-1576. CMP 2001:06

4. S. Baldwin and E. Slaminka, Calculating topological entropy, J. Stat. Phys. 89 (1997), 10171033. MR 99a:58100

5. L. Block and E. Coven, Approximating entropy of maps of the interval, Proceedings of the semester on Ergodic Theory and Dynamical Systems, 237-241, Banach Center Pub. 23, PWN, Warsaw, 1989. MR 92c:58106

6. R. Bowen, Entropy for group endomorphisms and homogeneous spaces, Trans. Am. Math. Soc. 153 (1971), 401-414 MR 43:469; erratum: Trans. Am. Math. Soc. 181 (1973), 509-510. MR 47:8810 
7. P. Góra and A. Boyarsky, Computing the topological entropy of general one-dimensional maps, Trans. Am. Math. Soc. 323 (1991), 39-49. MR 92a:58083

8. J. Llibre and M. Misiurewicz, Horseshoes, entropy and periods for graph maps, Topology 32 (1993), 649-664. MR 94k:58113

9. M. Misiurewicz and Z. Nitecki, Combinatorial patterns for maps of the interval, Mem. Amer. Math. Soc. 94, no. 456 (1991). MR 92h:58105

10. M. Misiurewicz and W. Szlenk, Entropy of piecewise monotone mappings, Studia Math. 67 (1980), 45-63. MR 82a:58030

11. S. Newhouse and T. Pignataro, On the estimation of topological entropy, J. Stat. Phys. 72 (1993), 1331-1351. MR 94i:58116

12. Y. Takahashi, A formula for topological entropy of one-dimensional dynamics, Sci. Papers College Gen. Ed. Univ. Tokyo 30 (1980), 11-22. MR 82i:58057

Departament de Matemàtiques, Edifici Cc, Universitat Autònoma de Barcelona, 08913 Cerdanyola del Vallès, Barcelona, Spain

E-mail address: alseda@mat.uab.es

Departament d'Informàtica i Matemàtica Aplicada, Universitat de Girona, Lluís SanTALÓ S/N, 17071 Girona, SpAin

E-mail address: juher@ima.udg.es

Departament de Matemàtica Aplicada i Anàlisi, Universitat de Barcelona, Gran Via 585, 08071 BARCELONA, SPAin

E-mail address: mumbru@mat.ub.es 